\title{
Dynamics of optical injection of charge and spin currents in quantum wells
}

\author{
D. H. Marti, M.-A. Dupertuis, and B. Deveaud \\ Institute of Quantum Electronics and Photonics, Ecole Polytechnique Fédérale de Lausanne (EPFL), CH-1015 Lausanne, Switzerland
}

(Received 28 May 2003; published 30 January 2004)

\begin{abstract}
We develop a dynamical theory for the optical injection of charge and spin current originating from the interferences between two coherent laser pulses of frequencies $\omega$ and $2 \omega$. Multiband Bloch equations which include one- and two-photon interband transitions are derived. They also account for ac Stark shifts and intersubband two-photon transitions. The model is used to describe the case of time-dependent charge and spin current injection in a symmetric GaAs/AlGaAs quantum well. A comparison to the bulk case is also made. The separate contributions of the populations and intersubband coherences to the charge current and $\mathrm{THz}$ emission are identified. The influence of the Stark shifts and the inter-valence-band two-photon transitions are calculated and discussed.
\end{abstract}

DOI: 10.1103/PhysRevB.69.035335

PACS number(s): 78.67.De, 78.20.Bh, 72.40.+w, 42.65.Sf

\section{INTRODUCTION}

In recent years, there has been considerable interest in coherent control of quantum-interference-related processes in semiconductors. It has been demonstrated that quantum interference between one- and two-photon absorption leads to measurable directional photocurrent in bulk for interband excitation $^{1,2}$ and in quantum wells (QW's) in the case of conduction-band to continuum transitions. ${ }^{3}$ Two coherent electromagnetic fields of frequency $\omega$ and $2 \omega$, with $\omega$ below and $2 \omega$ above the band gap, couple the same initial and final states and induce a polar asymmetry in the momentum space distribution of the carriers, which can be controlled by the relative phase of the beams. Such optical injection of current by femtosecond pulses has, for instance, been used for THzwave generation ${ }^{4}$ or proposed for demonstration of current echoes. ${ }^{5}$ With the same interference scheme, it is also possible to generate directional spin current, as shown by Bhat and Sipe, ${ }^{6}$ which can be of great interest in future spintronic applications. Optically injected charge current, which, according to the theory, should be spin polarized, has been measured in bulk GaAs. ${ }^{7}$ Recently, pure spin current (without any accompanying charge current) has been observed in bulk $^{8}$ and quantum wells. ${ }^{9}$ Current relaxation by scattering with LO phonons has also been included in the theoretical description by a Green's functions formalism. ${ }^{10}$ Finally, we note that research has now extended to materials other than GaAs, like GaN. ${ }^{11}$

To the best of our knowledge, all work published in this field concerns bulk semiconductors, excepted the early measurements by Dupont et al. ${ }^{3}$ who considered conductionband to continuum transitions in $\mathrm{AlGaAs} / \mathrm{GaAs}$ quantum well superlattices and the very recent measurement of pure spin current by Stevens et al. ${ }^{9}$ In the present work, we study the dynamics of charge and spin current injection by quantum interference between one- and two-photon interband transitions essentially for the case of quantum wells. The investigation of these processes in heterostructures is of great interest as the latter introduce new degrees of freedom and tunability. In order to study the temporal behavior, we derive specific multiband semiconductor Bloch equations, which go beyond Fermi's golden rule, used by most authors. Our model treats the whole dynamics of the lowest conduction band and the three highest valence bands, quasiresonantly coupled by two slowly varying monochromatic electromagnetic fields. The contribution of the far-off-resonant intermediate states to the second-order processes (Stark shift and two-photon transition) is taken into account within a perturbative approach and the carrier thermalization is included by phenomenological relaxation times. The calculation of the two-photon transitions requires intersubband momentum matrix elements, especially the band-diagonal part. ${ }^{12}$ They are obtained with a $\boldsymbol{k} \cdot \boldsymbol{p}$ envelope function method, ${ }^{13}$ together with the nonparabolic valence-band structure. We also calculate the far-field $\mathrm{THz}$ emission, taking into account the radiation from the charge current related to the coherences between the subbands, and show that they may contribute significantly to the emission. Finally, the influence of the ac Stark shifts and inter-valence-band (IVB) two-photon transitions on the charge current is evaluated and discussed.

The text is organized as follows. In Sec. II we start with a simple introduction to the core phenomenon of current generation by quantum interference between one- and twophoton transitions. Then we describe the quantum well model and develop the theory leading to multiband semiconductor Bloch equations including all the relevant terms. Section III is devoted to a numerical study and discussion of the results. Finally the conclusions are drawn in Sec. IV.

\section{THEORY}

\section{A. Quantum interferences between one- and two-photon transitions}

The interferences between one- and two-photon transitions can lead to different transition probabilities for states with opposite wave vectors $\boldsymbol{k}$ and $-\boldsymbol{k}$ (Fig. 1). This generates an asymmetric carrier distribution in reciprocal space resulting in a net charge and/or net spin current. In this section we give a short insight into the physics behind this process in the case of a centrosymmetric crystal by using simple grouptheoretical arguments. One has to emphasize that the spatial inversion symmetry is not mandatory for the subject dis- 


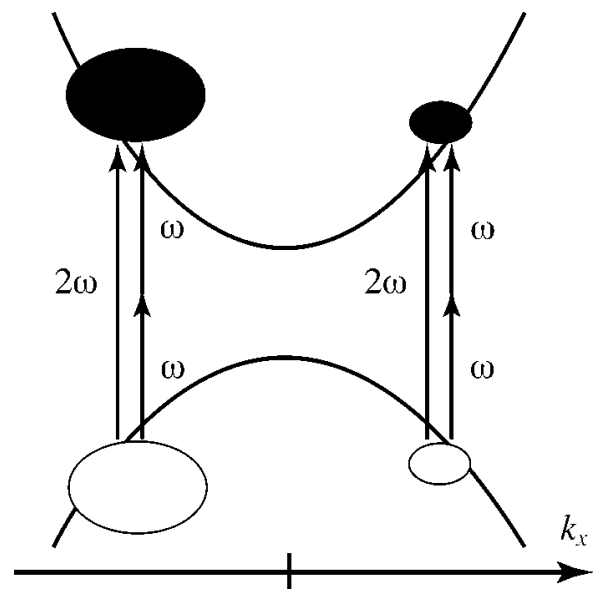

FIG. 1. Asymmetric interband excitation due to the interference between one- and two-photon transitions.

cussed here, but it is convenient. Together with time-reversal symmetry it implies that all energy bands are doubly degenerate (Appendix).

The dipole interaction $\boldsymbol{A} \cdot \boldsymbol{\Pi}$ between the carriers and electromagnetic fields is expressed in the velocity gauge where

$$
\boldsymbol{A}(t)=\boldsymbol{A}_{1} \cos \left(2 \omega t+\phi_{1}\right)+\boldsymbol{A}_{2} \cos \left(\omega t+\phi_{2}\right)
$$

is the vector potential describing the two monochromatic waves and

$$
\boldsymbol{\Pi}=\frac{-e}{m_{0}} \boldsymbol{\pi}=\frac{-e}{m_{0}}\left[\boldsymbol{p}+\frac{\hbar}{4 m_{0} c^{2}} \boldsymbol{\sigma} \wedge \nabla V\right]
$$

is proportional to the momentum operator $\boldsymbol{\pi}$.

For each pair of degenerate bands $(n, \bar{n})$, it is sufficient for our purpose to consider the four eigenstates of the Hamiltonian with same energy $|n,+\boldsymbol{k}\rangle,|\bar{n},+\boldsymbol{k}\rangle,|n,-\boldsymbol{k}\rangle$, and $|\bar{n},-\boldsymbol{k}\rangle$ (only two states are distinct for $\boldsymbol{k}=0$ ). They can be chosen so that (Appendix) the momentum matrix elements display the symmetry properties

$$
\begin{aligned}
& \boldsymbol{\Pi}_{m n,+\boldsymbol{k}}=-\boldsymbol{\Pi}_{m n,-\boldsymbol{k}}=+\boldsymbol{\Pi}_{\bar{n} \bar{m},+\boldsymbol{k}}=-\boldsymbol{\Pi}_{\bar{n} \bar{m},-\boldsymbol{k}}, \\
& \boldsymbol{\Pi}_{m \bar{n},+\boldsymbol{k}}=-\boldsymbol{\Pi}_{m \bar{n},-\boldsymbol{k}}=-\boldsymbol{\Pi}_{n \bar{m},+\boldsymbol{k}}=+\boldsymbol{\Pi}_{n \bar{m},-\boldsymbol{k}},
\end{aligned}
$$

between two states of same parity, and

$$
\begin{aligned}
& \boldsymbol{\Pi}_{m n,+\boldsymbol{k}}=+\boldsymbol{\Pi}_{m n,-\boldsymbol{k}}=+\boldsymbol{\Pi}_{\bar{n} \bar{m},+\boldsymbol{k}}=+\boldsymbol{\Pi}_{\bar{n} \bar{m},-\boldsymbol{k}}, \\
& \boldsymbol{\Pi}_{m \bar{n},+\boldsymbol{k}}=+\boldsymbol{\Pi}_{m \bar{n},-\boldsymbol{k}}=-\boldsymbol{\Pi}_{n \bar{m},+\boldsymbol{k}}=-\boldsymbol{\Pi}_{n \bar{m},-\boldsymbol{k}},
\end{aligned}
$$

between two states of opposite parity. Furthermore, for a quantum well oriented along a principal axis (e.g., [001]) the in-plane reflection symmetry ensures that $\boldsymbol{\epsilon}_{\perp} \cdot \boldsymbol{\Pi}_{m n, k}=0$ and $\boldsymbol{\epsilon}_{\|} \cdot \boldsymbol{\Pi}_{m \bar{n}, k}=0$ where $\boldsymbol{\epsilon}_{\perp}$ and $\boldsymbol{\epsilon}_{\|}$are, respectively, perpendicular and parallel to the quantum well. For an optical field polarized in the plane of the $\mathrm{QW}$, the $n \rightarrow \bar{m}$ transitions are therefore forbidden and we can restrict ourself to the cases $n \rightarrow m$ and $\bar{n} \rightarrow \bar{m}$.

We now consider resonant one- and two-photon transitions from two degenerate valence bands $v$ and $\bar{v}$ to two degenerate conduction bands $c$ and $\bar{c}$. The $v \rightarrow c$ transition amplitudes at wave vector $\boldsymbol{k}$ are given by $a_{1, k}=b_{1, k} e^{-i \phi_{1}}$ and $a_{2, k}=b_{2, k} e^{-i 2 \phi_{2}}$ with

$$
\begin{gathered}
b_{1, k}=(2 \hbar)^{-1} \sum_{\alpha} A_{1}^{\alpha} \Pi_{c v, k}^{\alpha}, \\
b_{2, k}=(2 \hbar)^{-2} \sum_{\alpha \beta} A_{2}^{\alpha} A_{2}^{\beta} \sum_{n} \frac{\Pi_{c n, k}^{\alpha} \Pi_{n v, k}^{\beta}}{\Omega_{c n}-\omega} .
\end{gathered}
$$

The sum $\Sigma_{n}$ is taken over all energy bands and $\Omega_{c n}$ is the interband frequency. The total transition probability is then given by $\left|a_{1, k}+a_{2, k}\right|^{2}=\left|a_{1, k}\right|^{2}+\left|a_{2, k}\right|^{2}+2 \operatorname{Re}\left[a_{1, k} * a_{2, k}\right]$. The first two terms on the right-hand side are phase independent and cannot generate asymmetric carrier distributions with respect to the inversion. The current is thus only injected by the interference term $I_{k}=2 \operatorname{Re}\left[a_{1, k} * a_{2, k}\right]$. The amplitudes $\bar{a}_{j, k}$ and $\bar{b}_{j, k}$ for the $\bar{v} \rightarrow \bar{c}$ transitions are obtained by replacing $c$ by $\bar{c}$ and $v$ by $\bar{v}$ in the above expressions. Let us define $\eta_{\boldsymbol{k}}=2 b_{1, \boldsymbol{k}} * b_{2, \boldsymbol{k}}$ and $\bar{\eta}_{\boldsymbol{k}}=2 \bar{b}_{1, \boldsymbol{k}} * \bar{b}_{2, \boldsymbol{k}}$ with the properties $\eta_{k}=-\eta_{-k}$ and $\eta_{k}=\bar{\eta}_{k}{ }^{*}$. The interference terms $I_{k}$ and $\bar{I}_{k}$ for the $v \rightarrow c$ and $\bar{v} \rightarrow \bar{c}$ transitions, respectively, then become

$$
\begin{aligned}
& I_{k}=\operatorname{Re}\left[\eta_{k}\right] \cos \left(2 \phi_{2}-\phi_{1}\right)+\operatorname{Im}\left[\eta_{k}\right] \sin \left(2 \phi_{2}-\phi_{1}\right), \\
& \bar{I}_{k}=\operatorname{Re}\left[\eta_{k}\right] \cos \left(2 \phi_{2}-\phi_{1}\right)-\operatorname{Im}\left[\eta_{k}\right] \sin \left(2 \phi_{2}-\phi_{1}\right)
\end{aligned}
$$

The velocity of an injected electron $|n, k\rangle$ is given by $-\Pi_{n n, k} / e$ and the expectation value of its spin perpendicular to the quantum well by $(\hbar / 2) \sigma_{n n, \boldsymbol{k}}=(\hbar / 2)\left\langle n, \boldsymbol{k}\left|\sigma_{z}\right| n, \boldsymbol{k}\right\rangle$, with symmetry properties

$$
\sigma_{n n, k}=\sigma_{n n,-k}=-\sigma_{\bar{n} \bar{n}, k}=-\sigma_{\bar{n} \bar{n},-k} .
$$

The contribution of the state $|n, k\rangle$ to the charge and spin current is given by $\boldsymbol{j}_{n, \boldsymbol{k}}=\boldsymbol{\Pi}_{n n, \boldsymbol{k}} I_{\boldsymbol{k}}$ and $\boldsymbol{s}_{n, \boldsymbol{k}}=-\sigma_{n n, \boldsymbol{k}} \boldsymbol{\Pi}_{n n, k} I_{\boldsymbol{k}}$, respectively. The spin has been multiplied by $2 e / \hbar$ to be expressed in the same units than the charge. The symmetry properties (3) and (5) involve $I_{k}=-I_{-k}$ and $\bar{I}_{k}=-\bar{I}_{-k}$. The net charge current $j$ and spin current $s$, obtained by summing over the four states in the same twice degenerate band, are thus given by

$$
\begin{aligned}
& j \propto 4 \operatorname{Re}\left[\eta_{k}\right] \cos \left(2 \phi_{2}-\phi_{1}\right), \\
& s \propto 4 \operatorname{Im}\left[\eta_{k}\right] \sin \left(2 \phi_{2}-\phi_{1}\right) .
\end{aligned}
$$

The total injected current is obtained by adding the contributions of all states. Yet each set of four resonantly excited states related by time reversal and spatial inversion will contribute in the same way to the current. Thus, Eqs. (12) and (13) show that the charge and spin currents are related, respectively, to the real and imaginary parts of the same tensor $\eta$ describing the interference between one- and two-photon transitions. Both current amplitudes can be controlled by the relative phase between the two electromagnetic waves, but not through the same dependence. Two linearly polarized 
beams can therefore be used to inject either pure charge or pure spin current by tuning the phase difference to 0 or $\pi / 2$, respectively.

In this paper we focus on the photocurrent generated by femtosecond pulses in quantum wells through interband transitions. In such structures, the inter-valence-band frequencies are comparable to the spectral width of the pulses, and one can no longer use a simple two-band model. As we shall see in the next sections, the intersubband coherences play a significant role in this study, especially concerning the $\mathrm{THz}$ emission. Furthermore, to obtain the right polarization dependence, one has to take into account the valence-band mixing.

In the following, we therefore derive adequate multiband semiconductor Bloch equations for the description of the optical current injection. They are applied to the specific case of a quantum well with realistic band structure, as well as to bulk GaAs. Yet the above model based on Fermi's golden rule highlights the underlying physical process, especially the phase dependence, which will be somewhat hidden in the Bloch equations.

\section{B. Quantum wells}

We consider a 70 - $\AA$-thick symmetric $\mathrm{GaAs} / \mathrm{Al}_{0.4} \mathrm{Ga}_{0.6} \mathrm{As}$ quantum well with growth direction along [001]. With this typical thickness, the QW has only a few (but more than one) valence subbands of confined states extending on a sufficiently wide range in reciprocal space. The inversion asymmetry in GaAs has very small effects on the valence-band structure and is therefore neglected (we recover a centrosymmetric case, justifying the Luttinger Hamiltonian for the description of the top of the valence band). This implies that the interferences between one- and two-photon transitions do not affect the total injected carrier density. Nevertheless, it has been demonstrated in GaAs (Ref. 14) that its asymmetry can be used to coherently control the carrier densities by a few percent.

The nonparabolic band structure and the momentum matrix elements for the optical transitions are calculated by a $\boldsymbol{k} \cdot \boldsymbol{p}$ envelope function method ${ }^{15}$ based on one doubly degenerate conduction band and the four-band Luttinger Hamiltonian. The eigenstates of the $\mathrm{QW}$ are described by the wave function $\Phi(\boldsymbol{r})=\Sigma_{a} F_{a}(z) U_{a}(\boldsymbol{r})$ where $U_{a}(\boldsymbol{r})$ are zonecenter Bloch functions of the bulk and $F_{a}(z)$ slowly varying envelope function along the confinement direction $z$. The sum is taken over a finite number of bulk states. We follow Burt's approach $^{13}$ and take the same basis $\left\{U_{a}\right\}$ for the whole heterostructure-i.e., the bulk states of GaAs (the QW). The functions $F_{a}$ are given by the eigensolutions of the effective Hamiltonian

$$
\begin{aligned}
\sum_{a^{\prime}}\{ & -\frac{\hbar^{2}}{2 m_{0}} \frac{d}{d z} \gamma_{a a^{\prime}}^{z z} \frac{d}{d z}-\frac{i \hbar^{2}}{2 m_{0}}\left[\frac{d}{d z} \gamma_{a a^{\prime}}^{z \mu}+\gamma_{a a^{\prime}}^{\mu z} \frac{d}{d z}\right] k_{\mu} \\
& -\frac{i \hbar}{m_{0}} P_{a a^{\prime}}^{z} \frac{d}{d z}+E_{a} \delta_{a a^{\prime}}+\frac{\hbar}{m_{0}} P_{a a^{\prime}}^{\mu} k_{\mu} \\
+ & \left.\frac{\hbar^{2}}{2 m_{0}} \gamma_{a a^{\prime}}^{\mu \nu} k_{\mu} k_{\nu}\right\} F_{a^{\prime}}(z)=E F_{a}(z),
\end{aligned}
$$

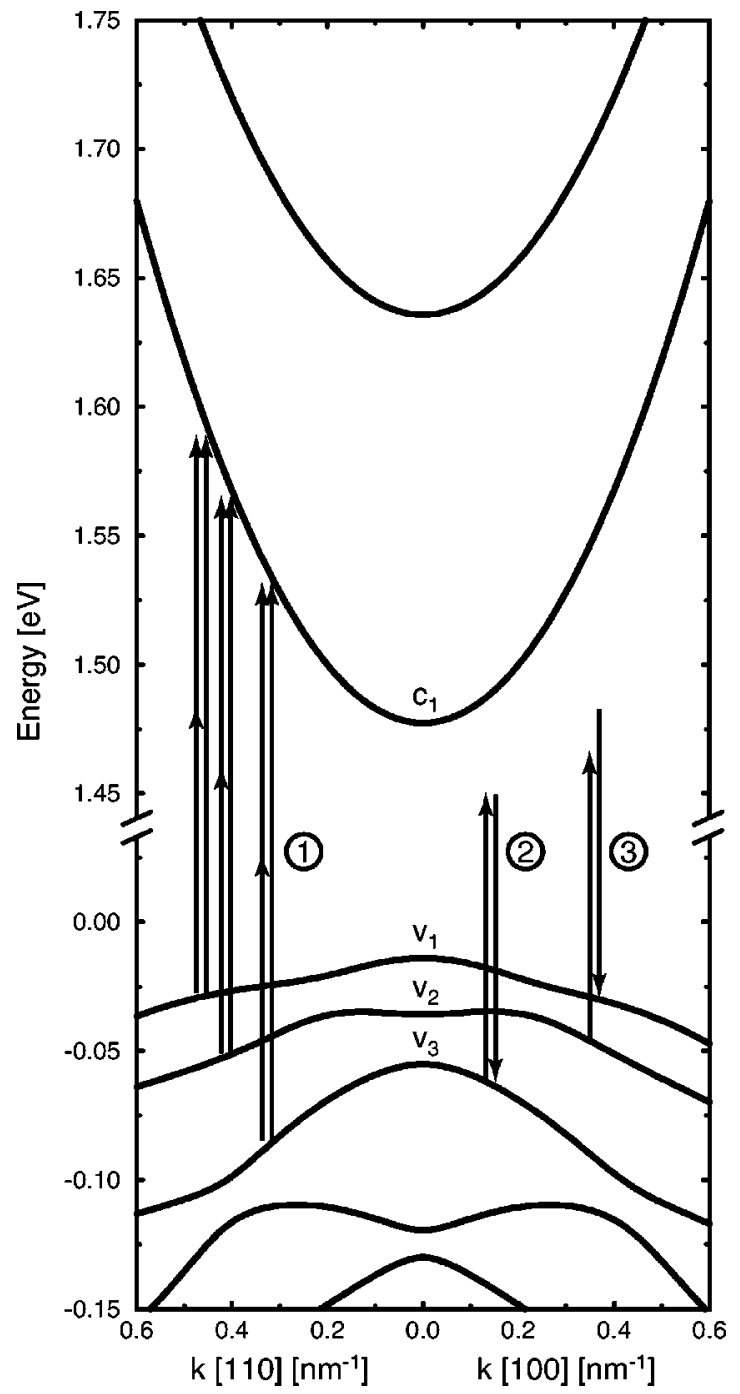

FIG. 2. Band structure of a $70-\AA$-thick $\mathrm{GaAs} / \mathrm{Al}_{0.4} \mathrm{Ga}_{0.6} \mathrm{As}$ quantum well used in the calculations (band gap: $1.491 \mathrm{eV}$ ). The arrows indicate the optical transitions included in the model: (1) One- and two-photon transitions between valence and conduction bands. (2) ac Stark shift. (3) Inter-valence-band two-photon transitions.

where $E_{a}$ is the energy of the Bloch state $U_{a}, P_{a a^{\prime}}$ the momentum matrix element between $U_{a}$ and $U_{a^{\prime}}$, and $\gamma$ the (dimensionless) inverse effective mass tensor. The repeated indices $\mu$ and $\nu$ are implicitly summed over the QW in-plane directions $x$ and $y$.

In the band structure model we use here, Eq. (14) does not couple the conduction bands to the valence bands and the two degenerate conduction bands are also independent. The effective Hamiltonian (14) reduces therefore to two (equivalent) one-dimensional equations for the conduction bands and the four-by-four Luttinger Hamiltonian for the valence bands.

Figure 2 shows the band structure used in this study and computed with the parameters ${ }^{16}$ given in Table I. Due to the time-reversal symmetry and the microscopic spatial inversion symmetry of the heterostructure, all subbands are twice degenerate.

In the following, we derive density matrix equations cor- 
TABLE I. Bulk band-structure parameters (Ref. 16) of $\mathrm{Al}_{x} \mathrm{Ga}_{1-x} \mathrm{As}$ used in this study. The energy gap is shifted to the room-temperature value.

\begin{tabular}{lccc}
\hline \hline Symbol & Parameter & $\mathrm{Al}_{x} \mathrm{Ga}_{1-x} \mathrm{As}$ & Unit \\
\hline$m_{c}$ & $\begin{array}{c}\text { Effective mass of the } \\
\text { conduction band }\end{array}$ & $0.0665+0.0835 x$ & {$\left[m_{0}\right]$} \\
& Luttinger parameter & $6.790-3.000 x$ & {$[-]$} \\
$\gamma_{1}$ & Luttinger parameter & $1.924-0.694 x$ & {$[-]$} \\
$\gamma_{2}$ & Luttinger parameter & $2.681-1.286 x$ & {$[-]$} \\
$\gamma_{3}$ & Kane parameter & 28.8 & {$[\mathrm{eV}]$} \\
$E_{P}$ & Energy gap & $1.423+1.247 x$ & {$[\mathrm{eV}]$} \\
$E_{g}$ & Band offsets & $68 / 32$ & {$[-]$} \\
$\Delta E_{c} / \Delta E_{v}$ & & & \\
\hline \hline
\end{tabular}

responding to the electronic states of the lowest conduction subband $c_{1}$ and the three highest valence subbands $v_{1}, v_{2}$, and $v_{3}$. All four bands are twice degenerate.

\section{Multiband semiconductor Bloch equations including two- photon transitions}

As a basis of the Hilbert space, we use the eigenstates of the quantum well Hamiltonian $H_{0}$ defined by

$$
H_{0}|n, \boldsymbol{k}\rangle=E_{n, k}|n, \boldsymbol{k}\rangle,
$$

where $n$ and $\boldsymbol{k}$ label, respectively, the energy band and the wave vector in the unconfined plane. $|n, \boldsymbol{k}\rangle$ denote the states found in Sec. II B. In the velocity gauge, the Hamiltonian for an electron interacting with a classical electromagnetic field is given by

$$
H=H_{0}+\frac{e}{m_{0}} \boldsymbol{A} \cdot \boldsymbol{\pi}+\frac{e^{2}}{2 m_{0}} \boldsymbol{A}^{2},
$$

where $\boldsymbol{A}$ is the classical vector potential and

$$
\boldsymbol{\pi}=\boldsymbol{p}+\frac{\hbar}{4 m_{0} c^{2}} \boldsymbol{\sigma} \wedge \nabla V
$$

is the momentum operator including the spin-orbit coupling. The elementary charge $e$ is positive. For optical frequencies, the momentum of the electromagnetic field can be neglected compared to the crystal momentum of the electrons. As a consequence, the spatial variation of the potential vector $\boldsymbol{A}$ can be ignored (dipole approximation) and only electronic states with equal wave vector are coupled by the electromagnetic interaction.

In the density matrix formalism, the Schrödinger equation can be written as

$$
\begin{aligned}
\partial_{t} \rho_{n n^{\prime}, k}= & -i \Omega_{n n^{\prime}, k} \rho_{n n^{\prime}, k}-\frac{i}{\hbar} \boldsymbol{A}(t) \sum_{m}\left[\rho_{n m, \boldsymbol{k}} \boldsymbol{\Pi}_{m n^{\prime}, \boldsymbol{k}}\right. \\
& \left.-\boldsymbol{\Pi}_{n m, \boldsymbol{k}} \boldsymbol{\rho}_{m n^{\prime}, \boldsymbol{k}}\right]
\end{aligned}
$$

where $n, n^{\prime}$, and $m$ label the energy bands and $\Omega_{n n^{\prime}, k}$ $=\left(E_{n, \boldsymbol{k}}-E_{n^{\prime}, \boldsymbol{k}}\right) / \hbar$ are the interband frequencies. The matrix II is defined by

$$
\boldsymbol{\Pi}_{n m, k}=\frac{-e}{m_{0}} \boldsymbol{\pi}_{n m, k},
$$

and the vector potential of the two quasimonochromatic fields is given by

$$
\boldsymbol{A}(t)=\boldsymbol{A}_{1}(t) \cos \left(\omega_{1} t+\phi_{1}\right)+\boldsymbol{A}_{2}(t) \cos \left(\omega_{2} t+\phi_{2}\right),
$$

with $\omega_{1}=2 \omega_{2}$. Note that the quadratic term $e^{2} A^{2} / 2 m_{0}$ in the Hamiltonian (16) does not contribute to the equation of motion (18), as it commutes with the density matrix.

We emphasize that the band-diagonal momentum matrix elements, which are usually neglected in the Bloch equations, are taken into account here since they give an important contribution to the interband two-photon transitions. ${ }^{12}$ The diagonal elements are in fact zero at zone center in a centrosymmetric crystal, but become large with increasing $\boldsymbol{k}$ vector.

Our aim is to derive effective time-dependent equations including all resonant one- and two-photon transitions between a limited number of energy bands. In a first step, the complete set of quantum states $\{n, \boldsymbol{k}\}$ is therefore restricted to the bands of interest to us. The withdrawn states are included in the equations as a perturbation up to first order in the inverse of the detuning between the corresponding interband frequency and the optical frequency. ${ }^{17}$ The transitions to the eliminated bands have thus to be far off resonant-i.e., with a large detuning - for the perturbative expansion to be valid. One is left with an effective Schrödinger equation for a reduced density matrix where the withdrawn bands appear as intermediate states in an effective interaction which is quadratic in the field (and linear in the inverse of the detuning). In a second step, we collect the part of the interaction giving rise to quasiresonant transitions and neglect the (small) offresonant contributions (generalized rotating wave approximation). This is best achieved in the rotating frame defined by the unitary transformation ${ }^{12} \bar{\rho}=U \rho U^{\dagger}$ where $U$ $=\exp (i \Lambda)$ with

$$
\Lambda_{m n, \boldsymbol{k}}(t)=\delta_{m n} \hbar^{-1}\left[E_{n, \boldsymbol{k}} t-\boldsymbol{\Pi}_{n n, \boldsymbol{k}} \int^{t} d t^{\prime} \boldsymbol{A}\left(t^{\prime}\right)\right]
$$

The right-hand side of the transformed equation of motion, $\partial_{t} \bar{\rho}=i\left[\bar{\rho}, U H U^{\dagger}\right]$, contains only driving terms which are linear or quadratic in the fields. The slowly varying linear driving terms (quasiresonant) give the dominant contribution to the one-photon transitions whereas the ones displaying rapid oscillations (off resonant) do not contribute significantly. As they may, however, be important for the resonant higherorder processes of interest to us (two-photon transitions, Stark shift, etc.), we cannot simply discard them. To treat these terms, we have developed a consistent scheme which amounts to using the same perturbative approach as in the first step where we eliminated the far-off-resonant bands and reduced the density matrix. As a result, we obtain an additional set of quasiresonant quadratic terms. This reflects the fact that these remaining bands participate also to higherorder transitions as intermediate states. Finally, among all the 
quadratic terms obtained, we retain consistently the slowly varying ones. The resulting Schrödinger equation is detailed below.

In the rotating frame, the time evolution of the reduced density matrix

$$
\begin{aligned}
\overline{\boldsymbol{\rho}}_{m n, \boldsymbol{k}}(t)= & \boldsymbol{\rho}_{m n, \boldsymbol{k}}(t) \exp \left[i \Omega_{m n, \boldsymbol{k}} t-i\left(\boldsymbol{\Pi}_{m m, \boldsymbol{k}}-\boldsymbol{\Pi}_{n n, \boldsymbol{k}}\right)\right. \\
& \left.\times \sum_{j=1,2} \frac{\boldsymbol{A}_{j}(t)}{\hbar \omega_{j}} \sin \left(\omega_{j} t+\boldsymbol{\phi}_{j}\right)\right]
\end{aligned}
$$

is now given by the following effective Schrödinger equation (multiband semiconductor Bloch equations including twophoton transitions)

$$
\partial_{t} \bar{\rho}_{k}=i\left[\bar{\rho}_{k}, H_{k}^{(1)}+H_{k}^{(2)}+H_{k}^{(\Delta)}\right] .
$$

The different quasiresonant transitions included in the effective Hamiltonian $H^{(1)}+H^{(2)}+H^{(\Delta)}$ are detailed below and sketched in Fig. 2. The considered states are divided into two sets: the conduction subbands and the valence subbands labeled by $c$ and $v$, respectively. The intersubband frequencies are far below the optical frequencies while the interband energies are close to them. The first two matrices

$$
H_{c v^{\prime}}^{(1)}=-(2 \hbar)^{-1} \sum_{\alpha} A_{1}^{\alpha} \Pi_{c v^{\prime}}^{\alpha} e^{i\left(\Omega_{c v^{\prime}}-\omega_{1}\right) t-i \phi_{1}}
$$

and

$$
\begin{aligned}
H_{c v^{\prime}}^{(2)}= & -(2 \hbar)^{-2} \sum_{\alpha, \beta} A_{2}^{\alpha} A_{2}^{\beta} e^{i\left(\Omega_{c v^{\prime}}-2 \omega_{2}\right) t-i 2 \phi_{2}} \\
& \times\left[\frac{1}{2} \sum_{n \neq c, v^{\prime}}\left(\frac{\Pi_{c n}^{\alpha} \Pi_{n v^{\prime}}^{\beta}}{\Omega_{n v^{\prime}}-\omega_{2}}-\frac{\Pi_{c n}^{\alpha} \Pi_{n v^{\prime}}^{\beta}}{\Omega_{c n}-\omega_{2}}\right)\right. \\
& \left.+\Pi_{c v^{\prime}}^{\alpha}\left(\Pi_{c c}^{\beta}-\Pi_{v^{\prime} v^{\prime}}^{\beta}\right) / \omega_{2}\right]
\end{aligned}
$$

describe, respectively, the one- and two-photon transitions between valence and conduction bands. The superscripts $\alpha$ and $\beta$ label the spatial directions $x, y$, and $z$. The off-diagonal terms of the third Hamiltonian matrix

$$
\begin{aligned}
H_{v v^{\prime}}^{(\Delta)}= & -(2 \hbar)^{-2} \sum_{\alpha, \beta} \sum_{j} A_{j}^{\alpha} A_{j}^{\beta} e^{i \Omega_{v v^{\prime \prime}}} \frac{1}{2} \\
& \times \sum_{\sigma= \pm 1} \sum_{n \neq v, v^{\prime}}\left[\frac{\Pi_{v n}^{\alpha} \Pi_{n v^{\prime}}^{\beta}}{\Omega_{n v}-\sigma \omega_{j}}+\frac{\Pi_{v n}^{\alpha} \Pi_{n v^{\prime}}^{\beta}}{\Omega_{n v^{\prime}}-\sigma \omega_{j}}\right]
\end{aligned}
$$

describe Raman-like intersubband two-photon transitions whereas the diagonal part contains the ac Stark shifts. $H_{c c^{\prime}}^{(\Delta)}$ is obtained by replacing $v$ and $v^{\prime}$ by $c$ and $c^{\prime}$ in the expression of $H_{v v^{\prime}}^{(\Delta)}$, and the remaining matrix elements $H_{c c^{\prime}}^{(1)}, H_{v v^{\prime}}^{(1)}$, $H_{c c^{\prime}}^{(2)}, H_{v v^{\prime}}^{(2)}, H_{v c^{\prime}}^{(\Delta)}$, and $H_{c v^{\prime}}^{(\Delta)}$ are all zero. For the sake of simplicity, the $\boldsymbol{k}$-vector indices have been omitted but should be added after each couple of band subscripts. In the above expressions, the sums over $n$ include all far-off-resonant bands eliminated from the equation of motion, as well as the bands of the reduced density matrix for which the corresponding denominator $\Omega \pm \omega_{j}$ is large. By contrast, the quadratic contributions from the transitions with small detuning $\Omega \pm \omega_{j}$ are automatically contained in the resonant linear interaction of $H_{c v^{\prime}}^{(1)}$. It must be pointed out that the perturbative approach described above does not preserve Hermiticity. This is related to the second-order transitions with energy mismatch, as is the case at most $\boldsymbol{k}$ vectors, for which the denominators $\left(\Omega_{n v}-\omega_{2}\right)$ and $-\left(\Omega_{c n}-\omega_{2}\right)$ are not equal. The departure from Hermiticity is of the order of the terms neglected when making the far-off-resonance approximation for the intermediate levels. Therefore it is justified to symmetrize the effective Hamiltonian as can be seen in Eqs. (25) and (26) (e.g., the factor $\frac{1}{2}$ ). A crucial benefit is to ensure accurately the total charge conservation in the crystal during the calculation. The decoherence and thermalization of the carriers are included through the phenomenological relaxation time $\tau$. The loss of coherence is thus modeled by

$$
\partial_{t} \bar{\rho}_{m n, k}=-\bar{\rho}_{m n, k} / \tau, \quad m \neq n,
$$

and the thermalization of the carrier distributions $n_{c, k}$ $=\bar{\rho}_{c c, \boldsymbol{k}}$ in the conduction bands and $n_{v, \boldsymbol{k}}=1-\bar{\rho}_{v v, \boldsymbol{k}}$ in the valence bands is described by

$$
\begin{aligned}
& \partial_{t} n_{c, \boldsymbol{k}}=-\left(n_{c, \boldsymbol{k}}-f_{c, \boldsymbol{k}}\right) / \tau, \\
& \partial_{t} n_{v, \boldsymbol{k}}=-\left(n_{v, \boldsymbol{k}}-f_{v, \boldsymbol{k}}\right) / \tau,
\end{aligned}
$$

where $f_{c / v, k}$ is the equilibrium Fermi distribution with timedependent carrier and energy densities given by the real instantaneous distribution $n_{c / v, k}$.

The effective equations (23) differ from the well-known semiconductor Bloch equations ${ }^{18,19}$ (SBE's) because we keep all intraband momentum matrix elements (including the band-diagonal ones) and all quasiresonant contributions of both frequencies. In the rotating frame, this was achieved by replacing the rapidly oscillating linear interaction terms by effective interactions which are quadratic in the field and eventually keeping only the slowly varying ones among them (generalized rotating-wave approximation). This is well justified close to resonance and leads to a Schrödinger equation with all interaction terms (one- and two-photon processes) varying on a same time scale (the pulse duration), which makes the numerical integration much more efficient. The microscopic Coulomb interaction is not included in our effective equations, but these additional terms would not differ from the ones appearing in the usual SBE's. It might, however, be a difficult task to work with them, especially for asymmetric carrier distributions as is the case for the present current injection.

\section{Polarization current}

The density of polarization current is obtained from the density matrix by $\boldsymbol{j}=(1 / S) \operatorname{Tr}[-e \boldsymbol{v} \rho]$, where the velocity $\boldsymbol{v}$ 
is related to the momentum $\boldsymbol{\pi}$ by $\boldsymbol{v}=(\boldsymbol{\pi}+e \boldsymbol{A}) / m_{0}$. By keeping the same notation, the density of current is therefore written as

$$
\boldsymbol{j}(t)=\frac{1}{S} \sum_{k} \sum_{n n^{\prime}}\left(\boldsymbol{\Pi}_{n n^{\prime}, \boldsymbol{k}}-\frac{e^{2}}{m_{0}} \boldsymbol{A}(t) \delta_{n n^{\prime}}\right) \boldsymbol{\rho}_{n^{\prime} n, \boldsymbol{k}}(t) .
$$

This expression has to be put in a form containing only the reduced density matrix-i.e., the bands for which the time evolution is calculated. This can be done by the same perturbative approach as above. Furthermore, in accordance with the approximations made to derive the effective multiband Bloch equations, the polarization current should also be calculated to the next order in the fields. Nevertheless, in this paper we focus on the "low-frequency" contribution whereas the higher-order terms in the current oscillate with frequencies around $\omega_{1}$ or $\omega_{2}$. The same applies to the contribution $\boldsymbol{A}(t) \rho_{n n, \boldsymbol{k}}$ in Eq. (30). We can therefore limit ourselves to $\boldsymbol{j}=(1 / S) \operatorname{Tr}[\boldsymbol{\Pi} \rho]$ where $\rho$ is restricted to the reduced density matrix.

The differentiation between charge and displacement current is somewhat arbitrary, as the whole polarization current is related to carrier movement. We will distinguish them in the frequency domain. Direct measurements of the electric current-e.g., through the voltage associated with charge accumulation ${ }^{2}$-are sensitive only to the low frequencies as the higher (optical) frequencies cannot be time resolved and vanish through the time integration. On the other hand, the high-frequency part of the current affects the optical properties of the medium and can be well described by optical susceptibilities. The low-frequency polarization current describing the injected charge current can therefore be written as

$$
\boldsymbol{j}=\frac{1}{S} \sum_{\boldsymbol{k}}\left[\sum_{c c^{\prime}} \boldsymbol{\Pi}_{c c^{\prime}, \boldsymbol{k}} \boldsymbol{\rho}_{c^{\prime} c, \boldsymbol{k}}+\sum_{v v^{\prime}} \boldsymbol{\Pi}_{v v^{\prime}, \boldsymbol{k}} \boldsymbol{\rho}_{v^{\prime} v, \boldsymbol{k}}\right] .
$$

In what follows, we distinguish between two contributions. First, the diagonal elements of $\rho$ (the populations) lead to the charge current $j_{\mathrm{pop}}=\Sigma_{n} j_{n}$ where

$$
\boldsymbol{j}_{n}=\frac{1}{S} \sum_{\boldsymbol{k}} \boldsymbol{\Pi}_{n n, \boldsymbol{k}} \rho_{n n, \boldsymbol{k}}
$$

is the current associated to the band $n$. The sum $\Sigma_{n}$ is taken over the conduction bands and valence bands. Second, the off-diagonal terms give rise to a charge current related to the intersubband coherences whose low-frequency part can be written as

$$
j_{\mathrm{coh}}=\frac{1}{S} \sum_{k}\left[\sum_{c \neq c^{\prime}} \boldsymbol{\Pi}_{c c^{\prime}, k} \rho_{c^{\prime} c, k}+\sum_{v \neq v^{\prime}} \boldsymbol{\Pi}_{v v^{\prime}, k} \boldsymbol{\rho}_{v^{\prime} v, k}\right] .
$$

The sums $\Sigma_{c \neq c^{\prime}}$ and $\Sigma_{v \neq v^{\prime}}$ are taken over all pairs of different conduction bands and valence bands, respectively. Note that the presence of coherence means that the quantum states of the carriers are not given by the occupation of the bands only.

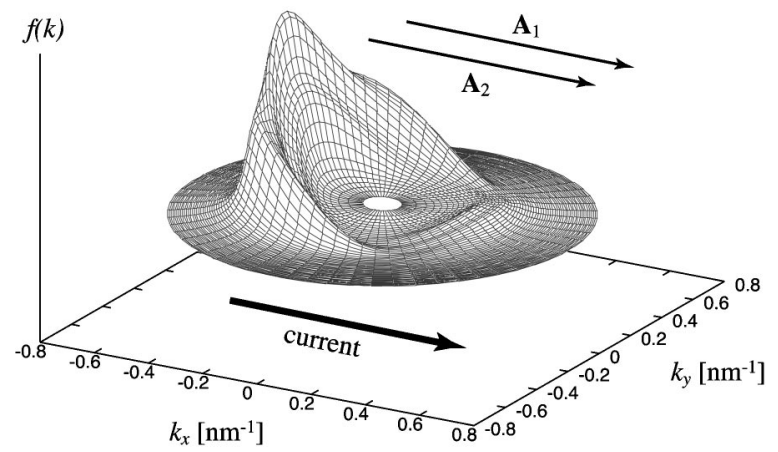

FIG. 3. Typical asymmetric carrier distribution in the conduction band of a QW, generated by two copolarized laser pulses with no phase difference $\left(2 \phi_{2}-\phi_{1}=0\right)$.

The theoretical works published up to now concern twoband models and/or focus on the electronic transition probabilities using Fermi's golden rule. Such models do not take into account intersubband coherences and give only access to the charge current associated with the carrier distribution in the band. But as mentioned above, the charge current which can be experimentally measured includes both contributions, and it will be shown in Sec. III that the part related to the coherences may indeed be important.

Note that the rapidly oscillating interband coherences $\rho_{c v}$ do not explicitly appear in the low-frequency part of the current but indeed contribute to the intersubband coherences and populations through the semiconductor Bloch equations.

One expects that the current related to the coherences oscillates with the intersubband frequencies and its contribution may therefore vanish in a time-integrated measurement. However, it also affects the electromagnetic radiation due to the variation in time of the current. Indeed, the power of the far-field $\mathrm{THz}$ emission originating from a finite volume $V$ is given by

$$
P(t)=\frac{1}{6 \pi \epsilon_{0} c^{3}}\left[\int_{V} \partial_{t}\left[j_{\mathrm{pop}}\left(\boldsymbol{r}^{\prime}, t\right)+j_{\mathrm{coh}}\left(\boldsymbol{r}^{\prime}, t\right)\right] d^{3} \boldsymbol{r}^{\prime}\right]^{2}
$$

and the corresponding power spectrum reads

$$
P(\nu) \propto\left|\int_{-\infty}^{+\infty} \partial_{t}\left(j_{\mathrm{pop}}+j_{\mathrm{coh}}\right) e^{i 2 \pi \nu t} d t\right|^{2} .
$$

Therefore, one can also distinguish between the two contributions $P=P_{\text {pop }}+P_{\text {coh }}$ related to the coherences and populations, respectively. As we will see, the intersubband coherences may significantly contribute to the radiated power.

By analogy to the charge current, we define the current of spins aligned along $\beta$ and moving along $\alpha$ as ${ }^{6}$

$$
s^{\alpha \beta}=\frac{1}{S} \operatorname{Tr}\left[(\hbar / 2) v^{\alpha} \sigma^{\beta} \rho\right],
$$

where $\sigma^{\beta}$ are the Pauli matrices and $v^{\alpha}$ the velocity operator. The superscripts $\alpha$ and $\beta$ denote Cartesian components. 

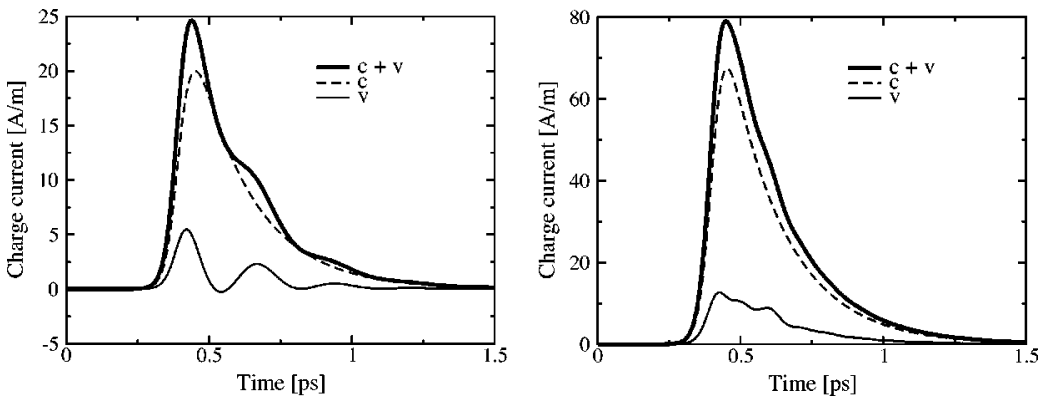

FIG. 4. Injected charge current along the onephoton polarization for $\hbar \omega_{1}=2 \hbar \omega_{2}=1.54 \mathrm{eV}$ (left) and $\hbar \omega_{1}=2 \hbar \omega_{2}=1.60 \mathrm{eV}$ (right). Thin lines: charge current in the conduction bands $(c)$ and in the valence bands $(v)$. Thick line: total charge current $(c+v)$.

\section{RESULTS}

\section{A. Introduction}

In the following, we discuss numerical results obtained with our model for two interfering femtosecond laser pulses. First, the optically injected charge current, related to the asymmetric carrier distribution in the bands (Fig. 3), and the corresponding far-field $\mathrm{THz}$ emission are calculated for the QW described in Sec. II B. Especially the contribution of the inter-valence-band coherences will be emphasized. We also give a quantitative comparison to bulk values. Then, using other beam polarizations, the generated pure spin current in the QW is computed. Finally, the influence of the ac Stark shifts and the IVB two-photon transitions are calculated and discussed.

For the calculations, eight energy bands have been used in the reduced density matrix: the lowest conduction band and the three highest valence bands, all of them being doubly degenerate. However, in order to estimate the contribution of the other bands shown in Fig. 2, we have also performed calculations where we included them (a) in the reduced density matrix or (b) as far off-resonant states (i.e., states that are not described by the reduced density matrix but nevertheless used as intermediate states in the quadratic interaction terms). First, it turns out that for the optical frequencies used in this study the neglected bands are almost not excited by the pulses and the results are not affected qualitatively. The corresponding interband transitions can thus be considered as off resonant. Second, the two-photon transition amplitude is modified when these additional bands are taken into account. Nevertheless, this increases the injected current by only $10 \%-15 \%$ in our typical conditions.

All subsequent results have been obtained with two 100-fs "sech" laser pulses polarized in the plane of the quantum well (TE mode). The peak intensities of the two pulses are chosen to lead separately to the same carrier injection in order to obtain a high contrast in the interference. Two pairs of frequencies have been used: 1.54/0.77 eV and 1.60/0.80 $\mathrm{eV}$ for the one-photon/two-photon beams, which corresponds to excitation $\sim 50 \mathrm{meV}$ and $\sim 110 \mathrm{meV}$ above the band gap of the quantum well $(1.491 \mathrm{eV})$. In both cases, the peak intensity of the two-photon pulse is taken to be $10 \mathrm{GW} / \mathrm{cm}^{2}$. The corresponding peak intensities of the one-photon pulse are $18 \mathrm{MW} / \mathrm{cm}^{2}$ and $42 \mathrm{MW} / \mathrm{cm}^{2}$ for the lower and higher frequencies, respectively. These values lead to injected carrier densities in the quantum well of $0.8 \times 10^{11} \mathrm{~cm}^{-2}$ and $1.6 \times 10^{11} \mathrm{~cm}^{-2}$.

The current decay is related to the thermalization of the carrier distributions and, more specifically, to the relaxation of the total crystal momentum in each band. Thus let us consider the two main mechanisms which govern electron scattering: emission of LO phonons and the carrier-carrier Coulomb interaction. The latter can further be divided into intraband (electron-electron) and interband (electron-hole) scattering. Král and Sipe ${ }^{10}$ already considered the emission of LO phonons in bulk GaAs for carriers excited at high energy (600 meV above the band gap) and obtained a current decay time of $50 \mathrm{fs}$. In the present work, the excitation energy is much lower (50 and $110 \mathrm{eV}$ above the band gap) and the corresponding relaxation is thus expected to be slower ${ }^{20}$ ( $\sim 200 \mathrm{fs}$ ). At high density, carrier-carrier scattering may, however, become dominant. But by contrast to the former mechanism, the Coulomb interaction conserves the total momentum of the carrier distribution. The fast intraband scattering alone is thus not sufficient for the current to vanish but needs the interplay with interband scattering and/or phonon emission. The usual thermalization times due to carriercarrier Coulomb interactions (about 100 fs and 1 ps for the intraband and interband contributions, respectively) are in general related to symmetric out-of-equilibrium distributions and may therefore not be appropriate in the context of current injection. A detailed description of the current decay is difficult because of the asymmetric distributions and we finally use the phenomenological relaxation time $\tau=200$ fs.
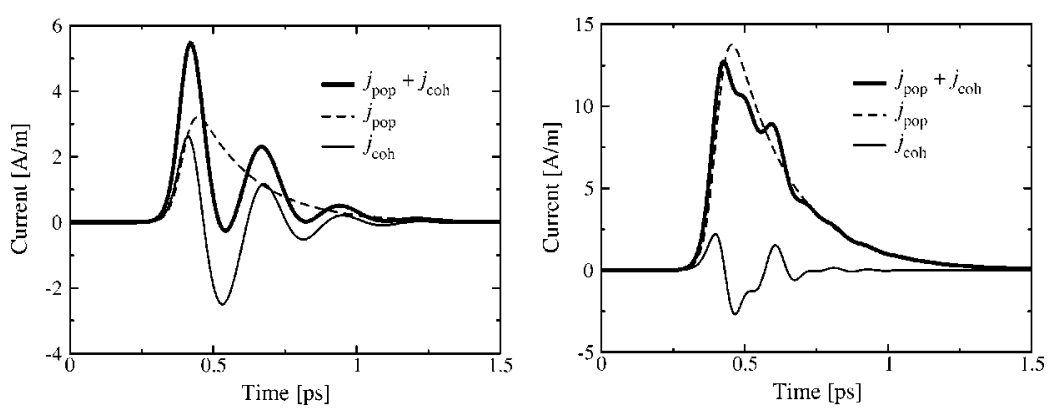

FIG. 5. Injected charge current in the valence bands along the one-photon polarization for $\hbar \omega_{1}=2 \hbar \omega_{2}=1.54 \mathrm{eV}$ (left) and $\hbar \omega_{1}=2 \hbar \omega_{2}$ $=1.60 \mathrm{eV}$ (right). Thick line: total charge current in the valence bands $\left(j=j_{\text {pop }}+j_{\text {coh }}\right)$. Thin lines: charge current related to the populations $\left(j_{\text {pop }}\right)$ and to the coherences $\left(j_{\text {coh }}\right)$. 

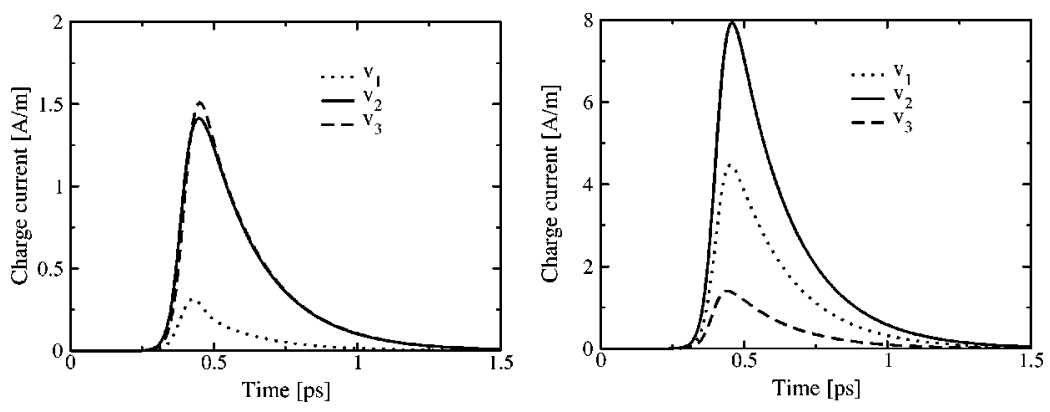

FIG. 6. Injected charge current $\boldsymbol{j}_{\text {pop }}$ related to the populations in the valence bands along the one-photon polarization for $\hbar \omega_{1}=2 \hbar \omega_{2}$ $=1.54 \mathrm{eV} \quad$ (left) and $\hbar \omega_{1}=2 \hbar \omega_{2}=1.60 \mathrm{eV}$ (right). For each band label $v_{1}, v_{2}$, and $v_{3}$, the current has been summed over the corresponding pair of degenerate subbands.
In this paper, we focus on the results obtained for two electromagnetic pulses with TE polarization. The charge current injected with one beam in TM polarization is more than one order of magnitude smaller, and there is even no current generated when both fields are polarized perpendicular to the QW.

\section{B. Charge current}

Figure 4 shows the calculated charge current burst $\boldsymbol{j}(t)$ [Eq. (31)] injected by beams copolarized along a principal axis and flowing parallel to the one-photon polarization (there is no current injection perpendicular to the polarizations). Notice that due to the lateral confinement of the quantum well, it corresponds to a current per length unit $(\mathrm{A} / \mathrm{m})$. The exponential decay is directly related to the phenomenological thermalization of the carrier distribution. As there is no low-frequency interband coherence (i.e., between conduction and valence bands), the charge current (thick line) can be separated into the currents in the conduction bands (thin dashed line) and in the valence bands (thin solid line). The main contribution comes from the carrier motion in the conduction band because of the higher (diagonal) intraband momentum matrix element-i.e., of the higher velocity of the electrons. The oscillation of the current in the valence bands is due to the intersubband coherences (Fig. 5). As discussed above, the charge current $\boldsymbol{j}$ can be divided into two parts $\boldsymbol{j}_{\text {pop }}$ and $\boldsymbol{j}_{\text {coh }}$ related to the populations and coherences, respectively. The oscillations appear only in $\boldsymbol{j}_{\text {coh }}$ and correspond to the intervalence-band frequencies.

It is interesting to note that only $\boldsymbol{j}_{\text {pop }}$ contributes to the time-integrated current that is usually measured. Indeed, for $1.54 \mathrm{eV} \quad(1.60 \mathrm{eV}), \quad \int \boldsymbol{j}_{\mathrm{coh}} d t$ represents only $0.54 \%$ $(-0.13 \%)$ of the total charge current and $3.9 \%(-0.76 \%)$ of the current in the valence bands. The negative sign for $1.60 \mathrm{eV}$ means that the time-integrated contribution of $\boldsymbol{j}_{\text {coh }}$ diminishes the total integrated current.
The charge current $\boldsymbol{j}_{\text {pop }}$ in the valence bands can further be divided into the current in the different subbands (Fig. 6). Their relative contributions depend on the photon energy because of the complicated dispersion of the momentum matrix elements due to the valence-band mixing.

The multiband Bloch equations include also correctly the saturation effects due to the time-evolving phase space filling. However, our results indicate that the high-field intensities $\left(10 \mathrm{GW} / \mathrm{cm}^{2}\right.$ for the two-photon pulse) used in this study are not sufficient for the saturation to become important, and the decoherence time is also too short to allow Rabi flopping.

\section{Comparison with current injection in bulk}

The calculated peak intensities of the density of current in the QW (25 A/m for $1.54 \mathrm{eV}$ and $79 \mathrm{~A} / \mathrm{m}$ for $1.60 \mathrm{eV}$ ) are certainly much higher than to be expected in a real measurement. Indeed, it has been shown ${ }^{21}$ that some effects which have been neglected like frequency chirp in the pulses or spatial variation of the phase due to the frequency dependence of the refractive index can strongly reduce the current injection. Yet it is useful to compare current intensities in QW's to bulk values obtained with the same model and in a similar configuration. The structure of the multiband Bloch equations (23) remains the same except that the $\boldsymbol{k}$ vectors become three dimensional. The electronic energies and dipole matrix elements for the bulk are the values corresponding to the (twice-degenerate) conduction band and to the four-valence-band Luttinger Hamiltonian used for the band structure calculation of the well. The density of current (30) relates to a volume unit (the surface $S$ is replaced by the crystal volume).

As the band gaps for the QW (1.491 eV) and bulk (1.423 $\mathrm{eV})$ are different, we do not compare the injected currents for equal beam frequencies, but for equal energy differences with respect to the band gap. Thus we calculate the current
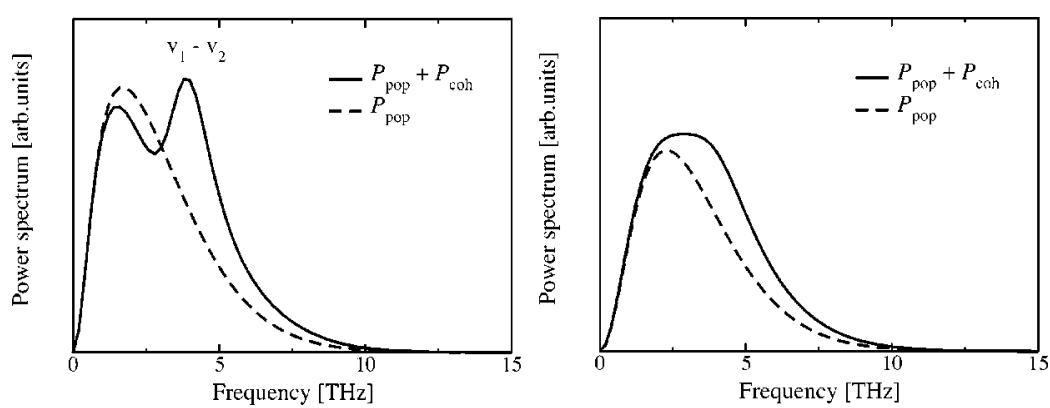

FIG. 7. Power spectrum of the far-field $\mathrm{THz}$ emission for $\hbar \omega_{1}=2 \hbar \omega_{2}=1.54 \mathrm{eV}$ calculated with two different thermalization and decoherence times: $\tau=200 \mathrm{fs}$ (left) and $\tau=100 \mathrm{fs}$ (right). Solid line: total THz emission $P=P_{\text {pop }}+P_{\text {coh }}$ due to the total charge current $\boldsymbol{j}=\boldsymbol{j}_{\text {pop }}+\boldsymbol{j}_{\text {coh }}$. Dashed line: THz emission $P_{\text {pop }}$ due to the charge current $\boldsymbol{j}_{\text {pop }}$ only. Both figures are plotted on the same scale. 

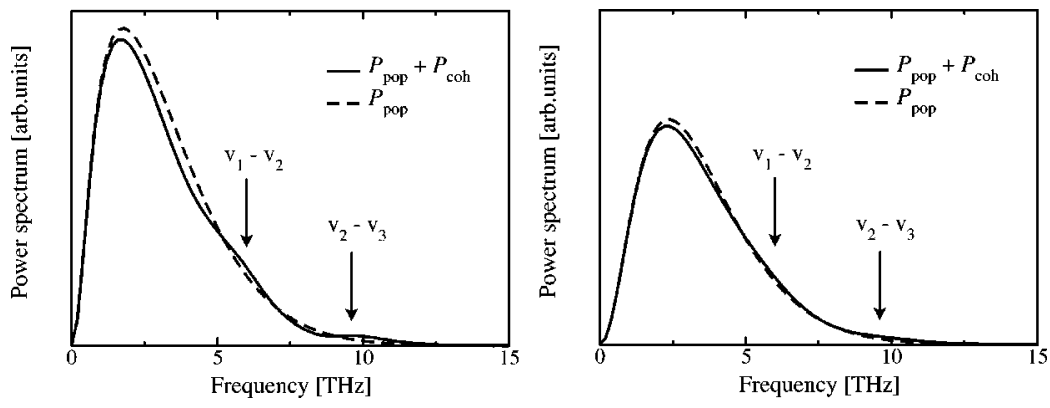

FIG. 8. Power spectrum of the far-field $\mathrm{THz}$ emission for $\hbar \omega_{1}=2 \hbar \omega_{2}=1.60 \mathrm{eV}$ calculated with two different thermalization and decoherence times: $\tau=200 \mathrm{fs}$ (left) and $\tau=100 \mathrm{fs}$ (right). Solid line: total THz emission $P=P_{\text {pop }}+P_{\text {coh }}$ due to the total charge current $\boldsymbol{j}=\boldsymbol{j}_{\mathrm{pop}}+\boldsymbol{j}_{\text {coh }}$. Dashed line: THz emission $P_{\text {pop }}$ due to the charge current $\boldsymbol{j}_{\text {pop }}$ only. Both figures are plotted on the same scale. generated in a GaAs bulk crystal by two pulses of frequency $1.47 \mathrm{eV}$ and $0.735 \mathrm{eV}$ (50 meV above the band gap) and copolarized along a principal axis. The peak intensities used of $16 \mathrm{MW} / \mathrm{cm}^{2}$ and $10 \mathrm{GW} / \mathrm{cm}^{2}$ for the one- and twophoton beams, respectively, excite independently a carrier density of $1.6 \times 10^{16} \mathrm{~cm}^{-3}$ in the conduction band. The model gives a maximal injected current of $1900 \mu \mathrm{A} / \mu \mathrm{m}^{2}$ in the bulk which is comparable to the $25 \mathrm{~A} / \mathrm{m}$ obtained in the QW for the frequency $1.54 \mathrm{eV}$ (also $50 \mathrm{meV}$ above the QW band gap). The bulk value is therefore comparable to the current expected by approximatively 77 QW's per $\mu \mathrm{m}$, insofar as the wells can be taken as independent. Indeed, these QW's would be separated by barriers of only $60 \AA$. We have to mention here that for numerical reasons the calculation for the bulk case has been done with a lower discretization of $\boldsymbol{k}$ space than for the quantum well, and the convergence has not been completely achieved.

\section{Far-field THz emission}

The power spectrum (35) of the far-field $\mathrm{THz}$ emission from the QW is plotted in Figs. 7 and 8 for photon energy $1.54 \mathrm{eV}$ and $1.60 \mathrm{eV}$, respectively. The dashed lines show the power $P_{\text {pop }}$ radiated only by the charge current pulse $j_{\text {pop }}$ whereas the solid lines represent the total $\mathrm{THz}$ emission $P$ $=P_{\text {pop }}+P_{\text {coh }}$ originating from $\boldsymbol{j}_{\text {pop }}+\boldsymbol{j}_{\text {coh }}$. One can clearly see the contributions from the field emitted by the current $\boldsymbol{j}_{\text {coh }}$ due to the intersubband coherences. The additional peaks around 5 and $10 \mathrm{THz}$ are related to the coherences between the pairs of valence subbands $v_{1}-v_{2}$ and $v_{2}-v_{3}$, respectively. The polarization between $v_{2}$ and $v_{3}$ for $1.54 \mathrm{eV}$ as well as between $v_{1}$ and $v_{3}$ for both energies is too small to be seen

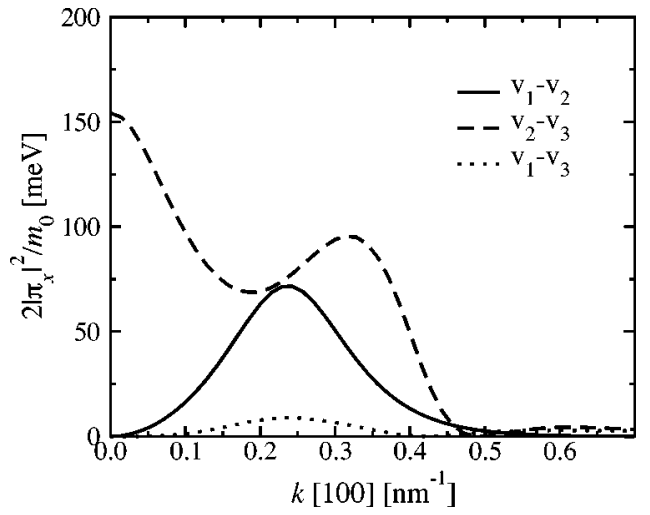

FIG. 9. Inter-valence-band momentum matrix elements $2\left|\pi_{x}\right|^{2} / m_{0}$. on this scale. The positions of these peaks are given by the intersubband frequencies and are therefore specific to the quantum well geometry. The radiated power for $1.60 \mathrm{eV}$ is about 10 times higher than for $1.54 \mathrm{eV}$ but the relative contribution of the inter-valence-band coherences to the total $\mathrm{THz}$ emission is much more important for the smaller injection energy. This can be explained by the dependence of the momentum matrix element between $v_{1}$ and $v_{2}$ on the wave vector $\boldsymbol{k}$ (Fig. 9). The lasers with frequency $1.54 / 0.77 \mathrm{eV}$ and $1.60 / 0.80 \mathrm{eV}$ excite mainly the states of the two highest valence bands with $\boldsymbol{k}$ vector around $0.25 \mathrm{~nm}^{-1}$ and $0.4 \mathrm{~nm}^{-1}$, respectively. The $v_{1}-v_{2}$ momentum matrix element is maximal in the first case and then decreases with increasing wave vector. The related polarization is expected to follow the same trend. On the other hand, the contribution $j_{\text {pop }}$ to the injected charge current is about 3-4 times higher at $1.60 \mathrm{eV}$ than at $1.54 \mathrm{eV}$. The related radiation $P_{\text {pop }}$ becomes therefore dominant at higher laser frequencies. Figure 7 also shows the effect of a shorter thermalization and decoherence time of $100 \mathrm{fs}$ on the spectrum. The contribution $P_{\text {pop }}$ from the populations is shifted to higher frequencies because of its faster decay and the different peaks of $P_{\text {coh }}$ are no longer well resolved.

It is important to emphasize that the low-frequency part of the current $\boldsymbol{j}_{\text {coh }}$ discussed above is also related to the interferences between the two fields, like the current $\boldsymbol{j}_{\text {pop }}$ generated by the asymmetric population distribution. Indeed, there is almost no $\mathrm{THz}$ emission when only one beam is present.

\section{E. Spin current}

As the spin current is also dominated by the carriers in the degenerate conduction band, we restrict ourself to these two bands. Furthermore, laser pulses polarized in the TE mode do not generate any coherence between bands $m$ and $\bar{n}$ which do not belong to the same irreducible representation ${ }^{1} E_{1 / 2}$ or ${ }^{2} E_{1 / 2}$, because the involved momentum matrix elements $\boldsymbol{\Pi}_{m \bar{n}}$ are equal to zero in the plane of the quantum well (Appendix). With the band structure model described in Sec. II $\mathrm{B}$, the spin current in the conduction bands therefore reduces to

$$
s^{z \beta}=-\frac{1}{S} \sum_{c} \sigma_{c c}^{z} \sum_{k} \Pi_{c c, k}^{\beta} \rho_{c c, k},
$$

where the left sum has to be taken over the two degenerate conduction bands with spin $\sigma=+1$ and $\sigma=-1$ pointing along the confinement direction $z$, and $s^{x \beta}=s^{y \beta}=s^{\alpha z}=0$. 

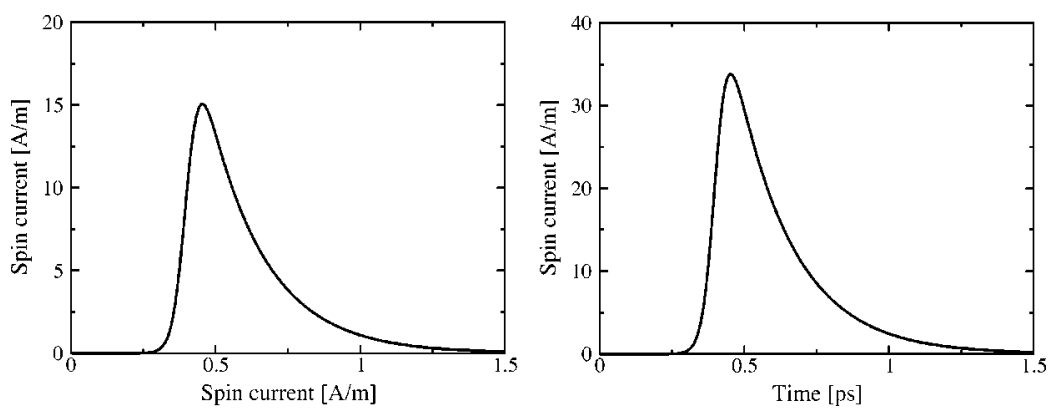

FIG. 10. Injected spin current in the conduction bands along the two-photon polarization for $\hbar \omega_{1}=2 \hbar \omega_{2}=1.54 \mathrm{eV}$ (left) and $\hbar \omega_{1}=2 \hbar \omega_{2}$ $=1.60 \mathrm{eV}$ (right).
The spin current has been expressed in the same units as the charge current and $\hbar / 2$ has therefore been replaced by $-e$ in Eq. (37).

The pure spin current (37) injected by cross-polarized laser beams and a phase difference of $\pi / 2$ is shown in Fig. 10 for the same frequencies and intensities as in the case of the charge current injection discussed above. The pulses are polarized along two principal axes and the spin current flows parallel to the two-photon polarization without any net charge flow. However, a small charge current due to the offresonant states subsists in the orthogonal in-plane direction (along the one-photon polarization). There is no $\mathrm{THz}$ emission polarized along the spin current direction and only a small radiation polarized perpendicularly to it (Fig. 11). The latter is essentially due to $\boldsymbol{j}_{\text {coh }}$ parallel to the one-photon polarization. The charge current and the $\mathrm{THz}$ emission are, respectively, about one and two orders of magnitude weaker than for the preceding configuration with copolarized beams and no phase difference.

\section{F. ac Stark shift and IVB transitions}

The multiband semiconductor Bloch equations (23) include all processes described by quasiresonant interaction terms up to second order in the EM fields, but only the interband transitions contribute directly to the current injection. The influence of the intersubband transitions on the charge current generation, however, remains an interesting issue.

Figure 12 displays the time-integrated charge current

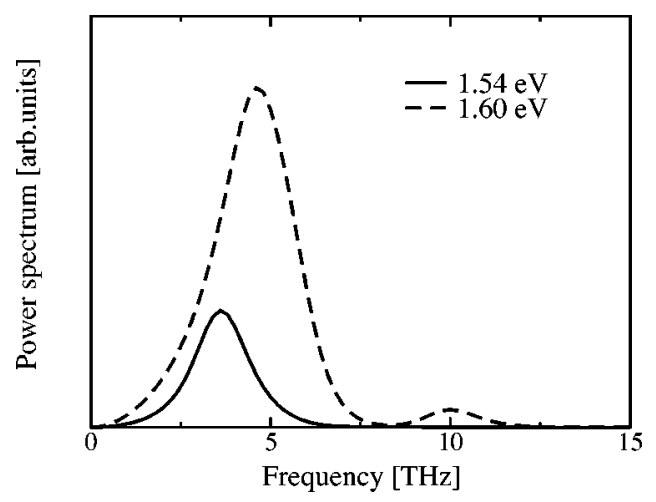

FIG. 11. Power spectrum of the far-field THz emission along the one-photon polarization in the configuration for spin current injection (cross-polarized beams with a phase difference of $\pi / 2$ ). Solid line: $\hbar \omega_{1}=2 \hbar \omega_{2}=1.54 \mathrm{eV}$. Dashed line: $\hbar \omega_{1}=2 \hbar \omega_{2}=1.60 \mathrm{eV}$. $\int \boldsymbol{j}(t) d t$ versus the photon energies in the cases where the Stark shift and/or the IVB transitions are neglected or not. The laser pulses are copolarized along one of the principal axis with no phase difference and constant peak intensities of $42 \mathrm{MW} / \mathrm{cm}^{2}$ and $10 \mathrm{GW} / \mathrm{cm}^{2}$ for the one- and two-photon beams, respectively. The ac Stark effect mainly shifts the current curve to higher energies in accordance with the fieldinduced band gap change. This results in weaker injected current for given optical frequencies, especially close to the band gap. At higher excitation energy, both computed currents become comparable because the ac Stark shift decreases for electronic states with higher $\boldsymbol{k}$ vector. The contribution of the IVB Raman-like two-photon transitions to the total charge current is rather weak. Indeed, they mainly affect the carrier distributions in the valence bands whereas the charge current is dominated by carriers in the conduction band.

\section{CONCLUSION}

We have developed a model of multiband Bloch equations including one- and two-photon interband transitions, the ac Stark shift, and inter-valence-band two-photon transitions. It has been used to calculate the charge and spin current injection and the corresponding $\mathrm{THz}$ emission in a symmetric semiconductor quantum well. This allows us to evidence that intersubband coherences are important for $\mathrm{THz}$ radiation (Figs. 7 and 8). We also discussed the influence of the Stark

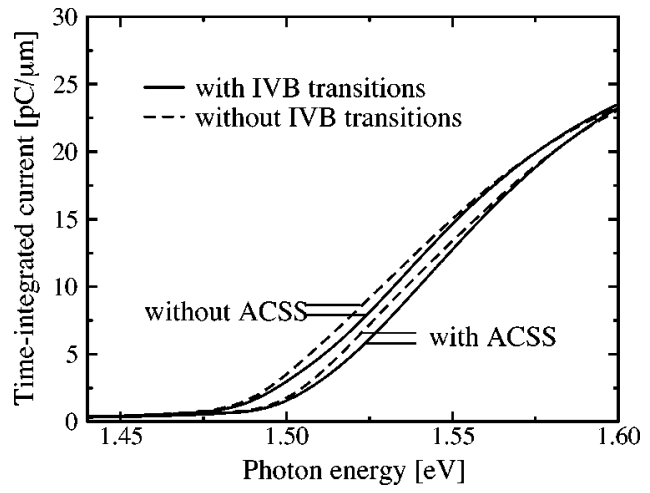

FIG. 12. Time-integrated charge current, function of the photon energy $\hbar \omega_{1}=2 \hbar \omega_{2}$ with and without the Stark shift and/or the IVB transitions. The two lower (upper) curves correspond to calculations with (without) the ac Stark shift (ACSS). For both of these pairs, the solid line includes the IVB transitions whereas for the dashed line they are neglected. Band gap of the quantum well: $1.491 \mathrm{eV}$. 
shift and IVB transitions on the current injection.

The decay of the current has been included in the model by the mean of a phenomenological relaxation time. A microscopic description of the thermalization of the carrier distributions would therefore be an important improvement. The interaction with LO phonons has already been considered by Král and Sipe. ${ }^{10}$ Intraband Coulomb scattering cannot directly cause the decay of the current, as it conserves the total crystal momentum in the band and its interband counterpart takes place on a longer time scale. Further work in the direction of including the Coulomb interaction, especially excitonic effects, is in progress.

\section{APPENDIX}

Let $K$ and $I$ be the antiunitary time-reversal operator and the unitary spatial inversion operator, respectively. For a system with half-integer spin, $K$ and $I$ have the properties $K^{2}$ $=-1$ and $I^{2}=1$.

Let $\psi_{k}$ be an eigenstate of the Hamiltonian $H(\boldsymbol{k})$ with energy $E_{\boldsymbol{k}}$ and wave vector $\boldsymbol{k}$. We show now that $K I \psi_{\boldsymbol{k}}$ is not only an eigenstate of $H(\boldsymbol{k})$ but also orthogonal to $\psi_{\boldsymbol{k}}$. In other words, the eigenspace of $H(\boldsymbol{k})$ associated with the energy $E_{\boldsymbol{k}}$ and wave vector $\boldsymbol{k}$ is at least of dimension 2. First, we note that the operator $K I$ is antiunitary with $(K I)^{2}$ $=-1$. Second we compute the scalar product

$$
\left\langle\psi_{k} \mid K I \psi_{k}\right\rangle=\left\langle(K I)^{2} \psi_{k} \mid K I \psi_{k}\right\rangle=-\left\langle\psi_{k} \mid K I \psi_{k}\right\rangle,
$$

where we have used the antiunitary property of $K I$. It follows that

$$
\left\langle\psi_{k} \mid K I \psi_{k}\right\rangle=0
$$

and the band structure is at least twice degenerate.

Let us now denote the two states with same energy and wave vector by $|n,+\boldsymbol{k}\rangle$ and $|\bar{n},+\boldsymbol{k}\rangle$ where $n$ and $\bar{n}$ label the two degenerate bands. The four eigenstates of the Hamiltonian with same energy $|n,+\boldsymbol{k}\rangle,|\bar{n},+\boldsymbol{k}\rangle,|n,-\boldsymbol{k}\rangle$, and $|\bar{n},-\boldsymbol{k}\rangle$ for $\boldsymbol{k} \neq 0$ are generally related to each other by

$$
\begin{aligned}
& I|n,+\boldsymbol{k}\rangle=+e^{i \phi}|n,-\boldsymbol{k}\rangle, \\
& I|\bar{n},+\boldsymbol{k}\rangle=+e^{i \bar{\phi}}|\bar{n},-\boldsymbol{k}\rangle, \\
& K|n,+\boldsymbol{k}\rangle=+e^{i \theta}|\bar{n},-\boldsymbol{k}\rangle, \\
& K|\bar{n},+\boldsymbol{k}\rangle=-e^{i \bar{\theta}}|n,-\boldsymbol{k}\rangle .
\end{aligned}
$$

One easily shows that

$$
(K I)^{2}|n,+\boldsymbol{k}\rangle=-e^{i(\phi+\theta-\bar{\phi}-\bar{\theta})}|n,+\boldsymbol{k}\rangle,
$$

and with $(K I)^{2}=-1$, one gets

$$
e^{i(\phi+\theta) / 2}= \pm e^{i(\bar{\phi}+\bar{\theta}) / 2} .
$$

By choosing the phases of the wave functions like

$$
\begin{aligned}
|n, \pm \boldsymbol{k}\rangle^{\prime} & =e^{i(\theta \overline{+} \phi) / 2}|n, \pm \boldsymbol{k}\rangle, \\
|\bar{n}, \pm \boldsymbol{k}\rangle^{\prime} & =e^{i(\bar{\theta} \overline{\overline{ }} \bar{\phi}) / 2}|\bar{n}, \pm \boldsymbol{k}\rangle,
\end{aligned}
$$

with

$$
e^{i(\phi+\theta) / 2}=+e^{i(\bar{\phi}+\bar{\theta}) / 2},
$$

for even wave functions, and

$$
\begin{aligned}
|n, \pm \boldsymbol{k}\rangle^{\prime} & =e^{i(\theta \overline{+} \phi \bar{\mp}) / 2}|n, \pm \boldsymbol{k}\rangle, \\
|\bar{n}, \pm \boldsymbol{k}\rangle^{\prime} & =e^{i(\bar{\theta} \overline{\bar{\phi}} \bar{\phi} \pi) / 2}|\bar{n}, \pm \boldsymbol{k}\rangle,
\end{aligned}
$$

with

$$
e^{i(\phi+\theta) / 2}=-e^{i(\bar{\phi}+\bar{\theta}) / 2},
$$

for odd wave functions, one finds, respectively,

$$
\begin{aligned}
& I|n,+\boldsymbol{k}\rangle^{\prime}=+|n,-\boldsymbol{k}\rangle^{\prime}, \\
& I|\bar{n},+\boldsymbol{k}\rangle^{\prime}=+|\bar{n},-\boldsymbol{k}\rangle^{\prime}, \\
& K|n,+\boldsymbol{k}\rangle^{\prime}=+|\bar{n},-\boldsymbol{k}\rangle^{\prime}, \\
& K|\bar{n},+\boldsymbol{k}\rangle^{\prime}=-|n,-\boldsymbol{k}\rangle^{\prime}
\end{aligned}
$$

and

$$
\begin{array}{r}
I|n,+\boldsymbol{k}\rangle^{\prime}=-|n,-\boldsymbol{k}\rangle^{\prime}, \\
I|\bar{n},+\boldsymbol{k}\rangle^{\prime}=-|\bar{n},-\boldsymbol{k}\rangle^{\prime}, \\
K|n,+\boldsymbol{k}\rangle^{\prime}=-|\bar{n},-\boldsymbol{k}\rangle^{\prime}, \\
K|\bar{n},+\boldsymbol{k}\rangle^{\prime}=+|n,-\boldsymbol{k}\rangle^{\prime} .
\end{array}
$$

The choice of Eqs. (A11) and (A14) ensures that $K I|n,+\boldsymbol{k}\rangle^{\prime}=|\bar{n},+\boldsymbol{k}\rangle^{\prime}$ in both cases. The symmetry relations $I \boldsymbol{\pi} I=-\boldsymbol{\pi}$ and $K \boldsymbol{\pi} K=-\boldsymbol{\pi}$ finally imply

$$
\begin{aligned}
& \boldsymbol{\Pi}_{m n,+\boldsymbol{k}}=-\boldsymbol{\Pi}_{m n,-\boldsymbol{k}}=+\boldsymbol{\Pi}_{\bar{n} \bar{m},+\boldsymbol{k}}=-\boldsymbol{\Pi}_{\bar{n} \bar{m},-\boldsymbol{k}}, \\
& \boldsymbol{\Pi}_{m \bar{n},+\boldsymbol{k}}=-\boldsymbol{\Pi}_{m \bar{n},-\boldsymbol{k}}=-\boldsymbol{\Pi}_{n \bar{m},+\boldsymbol{k}}=+\boldsymbol{\Pi}_{n \bar{m},-\boldsymbol{k}},
\end{aligned}
$$

for states of same parity, and

$$
\begin{aligned}
& \boldsymbol{\Pi}_{m n,+\boldsymbol{k}}=+\boldsymbol{\Pi}_{m n,-\boldsymbol{k}}=+\boldsymbol{\Pi}_{\bar{n} \bar{m},+\boldsymbol{k}}=+\boldsymbol{\Pi}_{\bar{n} \bar{m},-\boldsymbol{k}}, \\
& \boldsymbol{\Pi}_{m \bar{n},+\boldsymbol{k}}=+\boldsymbol{\Pi}_{m \bar{n},-\boldsymbol{k}}=-\boldsymbol{\Pi}_{n \bar{m},+\boldsymbol{k}}=-\boldsymbol{\Pi}_{n \bar{m},-\boldsymbol{k}},
\end{aligned}
$$

for states of opposite parity.

Note that the noncompulsory choice of different phases according to parity ensures that the above relations remain valid for $\boldsymbol{k}=0$. Indeed, Eqs. (A15) and (A19) must be seen as the definition of the band label $n$ in the two-dimensional subspace associated with $-\boldsymbol{k}$, with respect to $n$ in the subspace associated with $+\boldsymbol{k}$. This is not possible at zero wave vector as there are no more two distinct subspaces and the behavior under the inversion operation is dictated by parity.

For a quantum well orientated along a principal axis (e.g., [001]), the only symmetry which leaves $\boldsymbol{k}$ invariant besides the identity is the in-plane reflection. The little group of $\boldsymbol{k}$ is therefore $C_{S}$. Group-theory tables ${ }^{22}$ show that both irreducible representations (Irreps) ${ }^{1} E_{1 / 2}$ and ${ }^{2} E_{1 / 2}$ of the double group $C_{s}$ are one-dimensional and related to each other by 
time-reversal symmetry. In other words, the states labeled with the band index $n$ and $\bar{n}$ belong to ${ }^{1} E_{1 / 2}$ and ${ }^{2} E_{1 / 2}$, respectively. The momentum operators $\boldsymbol{\Pi}_{\|}$and $\boldsymbol{\Pi}_{\perp}$, parallel and perpendicular to the quantum well, belong, respectively, to the irreps $A^{\prime}$ and $A^{\prime \prime}$. Therefore, the representation products

$$
\begin{aligned}
& A^{\prime} \otimes{ }^{1} E_{1 / 2}={ }^{1} E_{1 / 2}, \quad A^{\prime \prime} \otimes{ }^{1} E_{1 / 2}={ }^{2} E_{1 / 2}, \\
& A^{\prime} \otimes{ }^{2} E_{1 / 2}={ }^{2} E_{1 / 2}, \quad A^{\prime \prime} \otimes{ }^{2} E_{1 / 2}={ }^{1} E_{1 / 2}
\end{aligned}
$$

imply that $\boldsymbol{\epsilon}_{\perp} \cdot \boldsymbol{\Pi}_{n m}=0$ and $\boldsymbol{\epsilon}_{\|} \cdot \boldsymbol{\Pi}_{n \bar{m}}=0$.
${ }^{1}$ R. Atanasov, A. Haché, J.L.P. Hughes, H.M. van Driel, and J.E. Sipe, Phys. Rev. Lett. 76, 1703 (1996).

${ }^{2}$ A. Haché, Y. Kostoulas, R. Atanasov, J.L.P. Hughes, J.E. Sipe, and H.M. van Driel, Phys. Rev. Lett. 78, 306 (1997).

${ }^{3}$ E. Dupont, P.B. Corkum, H.C. Liu, M. Buchanan, and Z.R. Wasilewski, Phys. Rev. Lett. 74, 3596 (1995).

${ }^{4}$ D. Côté, J.M. Fraser, M. DeCamp, P.H. Bucksbaum, and H.M. van Driel, Appl. Phys. Lett. 75, 3959 (1999).

${ }^{5}$ J. Stippler, C. Schlichenmaier, A. Knorr, T. Meier, M. Lindberg, P. Thomas, and S.W. Koch, Phys. Status Solidi B 221, 379 (2000).

${ }^{6}$ R.D.R. Bhat and J.E. Sipe, Phys. Rev. Lett. 85, 5432 (2000).

${ }^{7}$ M.J. Stevens, A.L. Smirl, R.D.R. Bhat, J.E. Sipe, and H.M. van Driel, J. Appl. Phys. 91, 4382 (2002).

${ }^{8}$ J. Hübner, W.W. Rühle, M. Klude, D. Hommel, R.D.R. Bhat, J.E. Sipe, and H.M. van Driel, Phys. Rev. Lett. 90, 216601 (2003).

${ }^{9}$ M.J. Stevens, A.L. Smirl, R.D.R. Bhat, A. Najmaie, J.E. Sipe, and H.M. van Driel, Phys. Rev. Lett. 90, 136603 (2003).

${ }^{10}$ P. Král and J.E. Sipe, Phys. Rev. B 61, 5381 (2000).

${ }^{11}$ H.M. van Driel, R.D.R. Bhat, D. Côté, N. Laman, J.E. Sipe, M.J. Stevens, and A.L. Smirl (unpublished).

${ }^{12}$ P.E. Selbmann, M.-A. Dupertuis, S. Haacke, T. Hessler, J.L.
Pleumeekers, and B. Deveaud, Phys. Status Solidi B 206, 219 (1998).

${ }^{13}$ M.G. Burt, J. Phys.: Condens. Matter 4, 6651 (1992).

${ }^{14}$ J.M. Fraser, A.I. Shkrebtii, J.E. Sipe, and H.M. van Driel, Phys. Rev. Lett. 83, 4192 (1999).

${ }^{15}$ G. Bastard, Wave Mechanics Applied to Semiconductor Heterostructures (Les Éditions de Physique, Les Ulis, 1990).

${ }^{16}$ L.W. Molenkamp, R. Eppenga, G.W. Thooft, P. Dawson, C.T. Foxon, and K.J. Moore, Phys. Rev. B 38, 4314 (1988).

${ }^{17}$ P.N. Butcher and D. Cotter, The Elements of Nonlinear Optics (Cambridge University Press, Cambridge, England, 1990).

${ }^{18}$ M. Lindberg and S.W. Koch, Phys. Rev. B 38, 3342 (1988).

${ }^{19}$ H. Haug and S.W. Koch, Quantum Theory of the Optical and Electronic Properties of Semiconductors (World Scientific, Singapore, 1990).

${ }^{20}$ A. Leitenstorfer, C. Fürst, A. Laubereau, and W. Kaiser, Phys. Rev. Lett. 76, 1545 (1996).

${ }^{21}$ A. Haché, J.E. Sipe, and H.M. van Driel, IEEE J. Quantum Electron. 34, 1144 (1998).

${ }^{22}$ S. L. Altmann and P. Herzig, Point-Group Theory Tables (Oxford University Press, New York, 1994). 